NASA/CR- $1948 \cdot 207793$

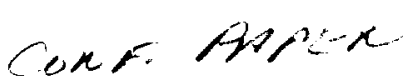

Draft paper for $34^{\text {th }}$ AIAA/ASME/SAE/ASEE Joint Propulsion Conference, July 13-15, 1998, Cleveland, Ohio

\title{
A GENERALIZED FLUID SYSTEM SIMULATION PROGRAM TO MODEL FLOW DISTRIBUTION IN FLUID NETWORKS
}

\author{
Alok Majumdar, John W. Bailey, Paul Schallhorn and Todd Steadman \\ Sverdrup Technology \\ Huntsville, Alabama - 35806
}

\begin{abstract}
This paper describes a general purpose computer program for analyzing steady state and transient flow in a complex network. The program is capable of modeling phase changes, compressibility, mixture thermodynamics and external body forces such as gravity and centrifugal. The program's preprocessor allows the user to interactively develop a fluid network simulation consisting of nodes and branches. Mass, energy and specie conservation equations are solved at the nodes; the momentum conservation equations are solved in the branches.

The program contains subroutines for computing "real fluid" thermodynamic and thermophysical properties for 33 fluids. The fluids are: helium, methane, neon, nitrogen, carbon monoxide, oxygen, argon, carbon dioxide, fluorine, hydrogen, parahydrogen, water, kerosene (RP-1), isobutane, butane, deuterium, ethane, ethylene, hydrogen sulfide, krypton, propane, xenon, R-11, R-12, R-22, R-32, R-123, R-124, R-125, R-134A, R$152 \mathrm{~A}$, nitrogen trifluoride and ammonia. The program also provides the options of using any incompressible fluid with constant density and viscosity or ideal gas.
\end{abstract}

Seventeen different resistance/source options are provided for modeling momentum sources or sinks in the branches. These options include: pipe flow, flow through a restriction, non-circular duct, pipe flow with entrance and/or exit losses, thin sharp orifice, thick orifice, square edge reduction, square edge expansion, rotating annular duct, rotating radial duct, labyrinth seal, parallel plates, common fittings and valves, pump characteristics, pump power, valve with a given loss coefficient, and a Joule-Thompson device.

The system of equations describing the fluid network is solved by a hybrid numerical method that is a combination of the Newton-Raphson and successive substitution methods. This paper also illustrates the application and verification of the code by comparison with Hardy Cross method for steady state flow and analytical solution for unsteady flow. 


\section{INTRODUCTION}

A fluid flow network consists of a group of flow branches, such as pipes and ducts, that are joined together at a number of nodes. They can range from simple systems consisting of a few nodes and branches to very complex networks containing many flow branches simulating valves, orifices, bends, pumps and turbines. In the analysis of existing or proposed networks, some node pressures and temperatures are specified or known. The problem is to determine all unknown nodal pressures, temperatures and branch flow rates.

This program requires that the flow network be resolved into nodes and branches. The program's preprocessor allows the user to interactively develop a fluid network simulation consisting of nodes and branches. In each branch, the momentum equation is solved to obtain the flow rate in that branch. At each node, the conservation of mass, energy and species equations are solved to obtain the pressures at that node.

The oldest method for systematically solving a problem consisting of steady flow in a pipe network is the Hardy Cross method [1]. Not only is this method suited for solutions generated by hand, but it has also been widely employed for use in computer generated solutions. But as computers allowed much larger networks to be analyzed, it became apparent that the convergence of the Hardy Cross method might be very slow or even fail to provide a solution in some cases. The main reason for this numerical difficulty is that

the Hardy Cross method does not solve the system of equations simultaneously. It considers a portion of the flow network to determine the continuity and momentum errors. The head loss and the flow rates are corrected and then it proceeds to an adjacent portion of the circuit. This process is continued until the whole circuit is completed. This sequence of operations is repeated until the continuity and momentum errors are minimized. It is evident that the Hardy Cross method belongs in the category of successive substitution methods and it is likely that it may encounter convergence difficulties for large circuits. In later years, the Newton-Raphson method has been utilized [2] to solve large networks, and with improvements in algorithms based on the Newton-Raphson method, computer storage requirements are not much larger than those needed by the Hardy Cross method.

The objective of the present effort is to develop: a) a robust and efficient numerical algorithm to solve a system of equations describing a flow network containing phase changes, mixing and rotation, and b) to implement the algorithm in a structured, easy-touse computer program. 


\section{MATHEMATICAL FORMULATION}

GFSSP assumes a Newtonian, steady, non-reacting and one dimensional flow in the flow circuit. The flow can be either laminar or turbulent, incompressible or compressible, with or without heat transfer, phase change and mixing. The analysis of the flow and pressure distribution in a complex fluid flow network requires resolution of the system into nodes and branches. Nodes can be either boundary nodes or internal nodes. Pressures, temperatures, and concentrations of fluid species are specified at the boundary nodes. At each internal node, scalar properties such as pressures, temperatures, enthalpies, and mixture concentrations are computed. The flow rates (vector properties) are computed at the branches.

\section{GOVERNING EQUATIONS}

Figure 1 displays a schematic showing adjacent nodes, their connecting branches, and the indexing system used by GFSSP. In order to solve for the unknown variables, mass, energy and fluid specie conservation equations are written for each internal node and flow rate equations are written for each branch.

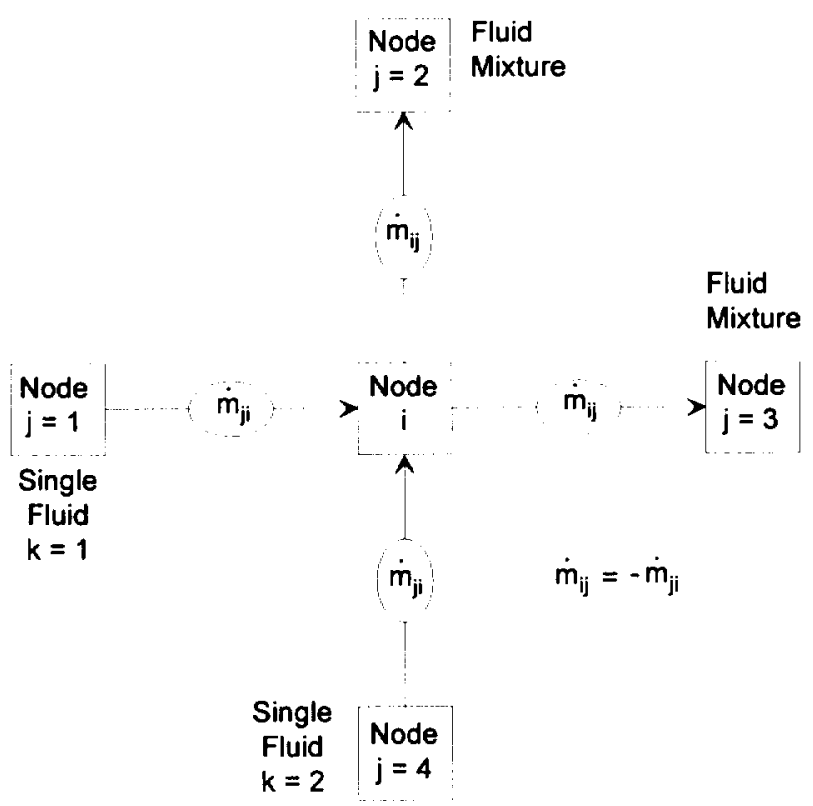

Figure 1 - Schematic of GFSSP Nodes, Branches and Indexing Practice

\section{Mass Conservation Equation}




$$
\frac{m_{\tau+\Delta \tau}-m_{\tau}}{\Delta \tau}=\sum_{j=1}^{j=n} m_{i j}
$$

Equation 1 requires that, for the transient formulation, the net mass flow from a given node must equate to rate of change of mass in the control volume. In the steady state formulation, the left hand side of the equation is zero. This implies that the total mass flow rate into a node is equal to the total mass flow rate out of the node.

\section{Momentum Conservation Equation}

The flow rate in a branch is calculated from the momentum conservation equation (Equation 2) which represents the balance of fluid forces acting on a given branch. A typical branch configuration is shown in Figure 2. Inertia, pressure, gravity, friction and centrifugal forces are considered in the conservation equation. In addition to these five forces, a source term $\mathrm{S}$ has been provided in the equation to input pump characteristics or to input power to a pump in a given branch. If a pump is located in a given branch, all other forces except pressure are set to zero. The source term, S, is set to zero in all branches without a pump.

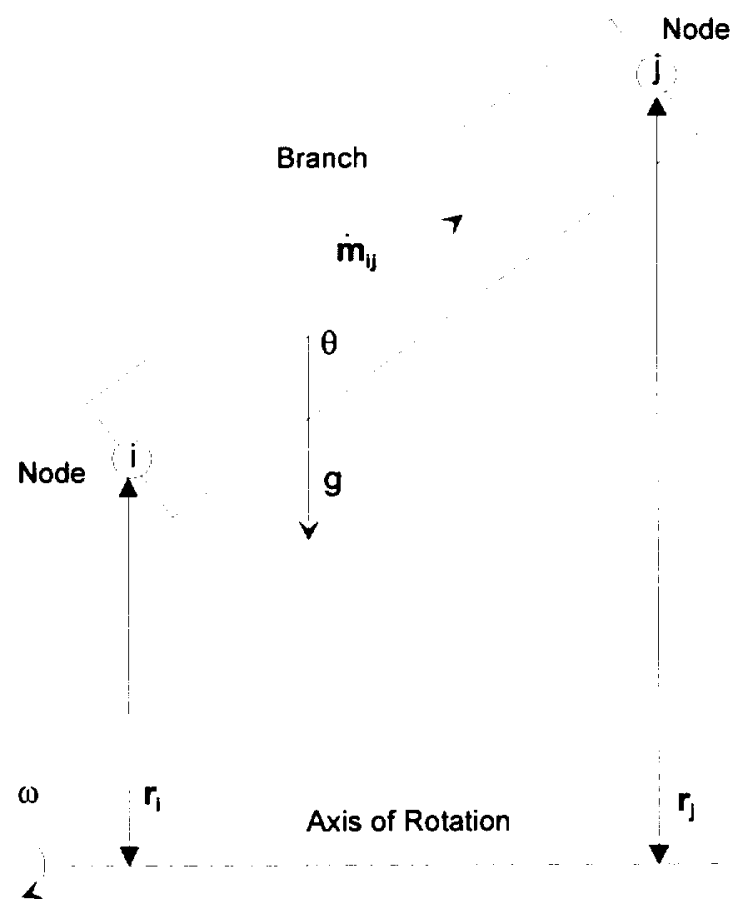

Figure 2 - Schematic of a Branch Showing Gravity and Rotation 


$$
\begin{aligned}
& \frac{\left(m u_{\tau+\Delta \tau}-m u_{\tau}\right)}{g_{c} \Delta \tau}+\frac{\dot{m}_{i j}}{g_{c}}\left(u_{i j}-u_{u}\right)= \\
& \left(p_{i}-p_{j}\right) A+\frac{\rho g V \cos \theta}{g_{c}}-K_{f} \dot{m}_{i j}\left|\dot{m}_{i j}\right| A+\frac{\rho K_{r o t}^{2} \omega^{2} A}{2 g_{c}}\left(r_{j}^{2}-r_{i}^{2}\right)+S
\end{aligned}
$$

The two terms in the left hand side of the momentum equation represent the inertia of the fluid. The first one is the time dependent term and must be considered for unsteady calculations. The second term is significant when there is a large change in area or density from branch to branch. The first term in the right hand side of the momentum equation represents the pressure gradient in the branch. The pressures are located at the upstream and downstream face of a branch. The second term represents the effect of gravity. The gravity vector makes an angle $(\theta)$ with the flow direction vector. The third term represents the frictional effect. Friction was modeled as a product of $\mathrm{K}_{\mathrm{r}}$ and the square of the flow rate and area. $K_{f}$ is a function of the fluid density in the branch and the nature of flow passage being modeled by the branch. The calculation of $\mathrm{K}_{\mathrm{f}}$ for different types of flow passages has been described in detail later within this report. The fourth term in the momentum equation represents the effect of the centrifugal force. This term will be present only when the branch is rotating as shown in Figure $2 . \mathrm{K}_{\mathrm{rot}}$ is the factor representing the fluid rotation. $\mathrm{K}_{\text {rot }}$ is unity when the fluid and the surrounding solid surface rotates with the same speed. This term also requires a knowledge of the distances between the upstream and downstream faces of the branch from the axis of rotation.

\section{Energy Conservation Equation}

The energy conservation equation for node i, shown in Figure 1, can be expressed mathematically as shown in Equation 3.

$$
\begin{aligned}
& \frac{m\left(h-\frac{p}{\rho J}\right)_{\tau+\Delta \tau}-m\left(h-\frac{p}{\rho J}\right)_{\tau}}{\Delta \tau}= \\
& \sum_{j=1}^{j=n}\left\{\operatorname{MAX}\left[-\dot{m}_{i j}, 0\right] h_{j}-\operatorname{MAX}\left[\dot{m}_{i j}, 0\right] h_{i}\right\}+\frac{\operatorname{MAX}\left[-\dot{m}_{i j}, 0\right]}{\left|\dot{m}_{i j}\right|}\left[\left(p_{i}-p_{j}\right)+K_{i j} \dot{m}_{i j}^{2}\right]+Q_{i}=0
\end{aligned}
$$

Equation 3 shows that for transient flow, the rate of increase of internal energy in the control volume is equal to the rate of energy transport into the control volume minus the rate of energy transport from the control volume plus the rate of work done on the fluid by the pressure force plus the rate of work done on the fluid by the viscous force plus the rate of heat transfer into the control volume. 
For a steady state situation, the energy conservation equation, Equation 3, states that the net energy flow from a given node must equate to zero. In other words, the total energy leaving a node is equal to the total energy coming into the node from neighboring nodes and from any external heat sources $\left(Q_{i}\right)$ coming into the node and work done on the fluid by pressure and viscous forces. The MAX operator used in Equation 3 is known as an upwind differencing scheme which has been extensively employed in the numerical solution of Navier-Stokes equations in convective heat transfer and fluid flow applications [3]. When the flow direction is not known, this operator allows the transport of energy only from its upstream neighbor. In other words, the upstream neighbor influences its downstream neighbor but not vice versa. The second term in the right hand side represents the work done on the fluid by the pressure and viscous force. The difference between the steady and unsteady formulation lies in the left hand side of the equation. For a steady state situation, the left hand side of Equation 3 is zero, where as in unsteady cases the left hand side of the equation must be evaluated.

\section{Fluid Specie Conservation Equation}

The flow network shown in Figure 1 has a fluid mixture flowing in most of the branches. In order to calculate the density of the mixture, the concentration of the individual fluid species within the branch must be determined. The concentration for the $\mathrm{k}^{\text {th }}$ specie can be written as

$$
\frac{\left(m_{i} c_{i, k}\right)_{\tau+\Delta \tau}-\left(m_{i} c_{i, k}\right)_{\tau}}{\Delta \tau}=\sum_{j=1}^{j}\left\{\operatorname{MAX}\left[-\dot{m}_{i j}, 0\right] c_{j, k}-\operatorname{MAX}\left[\dot{m}_{i j}, 0\right] c_{i, k}\right\}
$$

For a transient flow, Equation 4, states that the rate of increase of the concentration of the $\mathrm{k}^{\text {th }}$ specie in the control volume equals the rate of transport of the $\mathrm{k}^{\text {th }}$ specie into the control volume minus the rate of transport of the $\mathrm{k}^{\text {th }}$ specie out of the control volume.

Like Equation 3, for steady state conditions, Equation 4 requires that the net mass flow of the $\mathrm{k}^{\text {th }}$ specie from a given node must equate to zero. In other words, the total mass flow rate of the given specie into a node is equal to the total mass flow rate of the same specie out of that node. For steady state, the left hand side of Equation 4 is zero. For the unsteady formulation, the resident mass in the control volume is changing and therefore, the left hand side must be computed.

\section{Thermodynamic and Thermophysical Properties}

The momentum conservation equation, Equation 2, requires knowledge of the density and the viscosity of the fluid within the branch. These properties are functions of the temperatures, pressures and concentrations of fluid species for a mixture. Three 
thermodynamic property routines have been integrated into the program to provide the required fluid property data. GASP [4] provides the thermodynamic and transport properties for ten fluids. These fluids include Hydrogen, Oxygen, Helium, Nitrogen, Methane, Carbon Dioxide, Carbon Monoxide, Argon, Neon and Fluorine. WASP [5] provides the thermodynamic and transport properties for water and steam. GASPAK [6] provides thermodynamic properties for helium, methane, neon, nitrogen, carbon monoxide, oxygen, argon, carbon dioxide, hydrogen, parahydrogen, water, isobutane, butane, deuterium, ethane, ethylene, hydrogen sulfide, krypton, propane, xenon, R-11, R12, R-22, R-32, R-123, R-124, R-125, R-134A, R-152A, nitrogen trifluoride and ammonia. For RP-1 fuel, a look up table of properties has been generated by a modified version of GASP. An interpolation routine has been developed to extract the required properties from the tabulated data.

\section{Friction Calculations}

It was mentioned earlier in this paper that the friction term in the momentum equation is expressed as a product of $\mathrm{K}_{\mathrm{f}}$, the square of the flow rate and the flow area. Empirical information is necessary to estimate $K_{f}$. Several options for flow passage resistance are listed in Table 1.

Table 1 - Resistance Options in GFSSP

\begin{tabular}{|c|c|c|c|c|c|}
\hline Option & Type of Resistance & Input Parameters & Option & Type of Resistance & Input Parameters \\
\hline 1 & Pipe Flow & $\begin{array}{l}\text { L (in), D (in), } \\
\varepsilon / \mathrm{D}\end{array}$ & 10 & $\begin{array}{c}\text { Rotating Radial } \\
\text { Duct }\end{array}$ & $\begin{array}{l}\text { L (in), D (in), } \\
\text { N (rpm) }\end{array}$ \\
\hline 2 & $\begin{array}{l}\text { Flow Through } \\
\text { Restriction }\end{array}$ & $C_{L}, A\left(\right.$ in $\left.^{2}\right)$ & 11 & Labyrinth Seal & $\begin{array}{l}\mathrm{r}_{\mathrm{i}} \text { (in), } \mathrm{c} \text { (in), } \mathrm{m} \\
\text { (in), } \mathrm{n}, \alpha\end{array}$ \\
\hline 3 & Non-circular Duct & $a$ (in), $b$ (in) & 12 & $\begin{array}{l}\text { Flow Between } \\
\text { Parallel Plates }\end{array}$ & $\begin{array}{c}r_{i}(\text { in), } c \text { (in), } \\
\text { L (in) }\end{array}$ \\
\hline 4 & $\begin{array}{l}\text { Pipe with Entrance } \\
\text { and Exit Loss }\end{array}$ & $\begin{array}{c}\mathrm{L} \text { (in), D (in), } \\
\varepsilon / \mathrm{D}, \mathrm{K}_{\mathrm{i}}, \mathrm{K}_{\mathrm{e}}\end{array}$ & 13 & $\begin{array}{c}\text { Common Fittings } \\
\text { and Valves (Two K } \\
\text { Method) }\end{array}$ & $\mathrm{D}$ (in), $\mathrm{K}_{1}, \mathrm{~K}_{2}$ \\
\hline 5 & Thin, Sharp Orifice & $\mathrm{D}_{1}$ (in), $\mathrm{D}_{2}$ (in) & 14 & $\begin{array}{c}\text { Pump } \\
\text { Characteristics }^{1}\end{array}$ & $\mathrm{~A}_{0}, \mathrm{~B}_{0}, \mathrm{~A}\left(\mathrm{in}^{2}\right)$ \\
\hline 6 & Thick orifice & $\begin{array}{c}\mathrm{L} \text { (in), } \mathrm{D}_{1} \text { (in), } \\
\mathrm{D}_{2} \text { (in) }\end{array}$ & 15 & Pump Power & $\mathrm{P}(\mathrm{hp}), \eta, \mathrm{A}\left(\mathrm{in}^{2}\right)$ \\
\hline 7 & Square Reduction & $\mathrm{D}_{1}$ (in), $\mathrm{D}_{2}$ (in) & 16 & $\begin{array}{c}\text { Valve with Given } \\
C_{\mathrm{v}}\end{array}$ & $C_{v}, A$ \\
\hline 8 & Square Expansion & $\mathrm{D}_{1}$ (in), $\mathrm{D}_{2}$ (in) & 17 & $\begin{array}{c}\text { Joule-Thompson } \\
\text { Device }\end{array}$ & $\mathrm{L}_{\mathrm{ohm}}, \mathrm{V}_{\mathrm{f}}, \mathrm{k}_{\mathrm{v}}, \mathrm{A}$ \\
\hline 9 & $\begin{array}{c}\text { Rotating Annular } \\
\text { Duct }\end{array}$ & $\begin{array}{c}\mathrm{L}(\text { in }), \mathrm{r}_{\mathrm{o}} \text { (in), } \\
\mathrm{r}_{\mathrm{i}} \text { (in), N (rpm) }\end{array}$ & & & \\
\hline
\end{tabular}


${ }^{1}$ Pump characteristics are expressed as $\Delta p=\mathrm{A}_{0}+\mathrm{B}_{0} \dot{m}^{2}$
$\Delta p-$ Pressure rise, $\mathrm{lbf} / \mathrm{ft}^{2}$
$\dot{m}-$ Flow rate, $\mathrm{lbm} / \mathrm{sec}$

\section{COMPUTER PROGRAM}

GFSSP was developed on an IBM compatible PC using the Lahey EM32 FORTRAN compiler. The same source code also runs on a Silicon Graphics workstation. The code was developed with a modular structure to achieve two objectives. First, the code's solver module was separated from the preprocessor module such that users are not required to write any code to develop their models. Secondly, the code can easily be extended to enhance its capability by adding new modules. The main routine controls all of the program operations and makes the decisions whether to continue or to stop the calculations.

The computer program has three major parts. The first part consists of the subroutines for the preprocessor. The preprocessor allows the user to interactively create the flow network model consisting of nodes and branches. All of the input specifications, including the boundary conditions, are specified through the preprocessor. The second major part of the program consists of the subroutines that provide the initial conditions and then develop and solve all of the conservation equations in the flow network. The third part of the program consists of the thermodynamic property programs, GASP, WASP, and GASPAK that provide the necessary thermodynamic and thermophysical property data required to solve the resulting system of equations.

Figure 3 shows GFSSP's process flow diagram. The user runs the interactive preprocessor to generate the input data file. The input data file contains all the information necessary for the model. The solver module reads the input data file and produces the solution in conjunction with the thermodynamic property programs. It should be noted that the user interfaces with the program only through the input data file. The user is never required to modify the source program to develop the model. The user is also not required to understand the structure of the solver module in order to develop their model. However, for the completeness of the documentation, a flow chart showing the major activities of the code is described in Figure 4. 


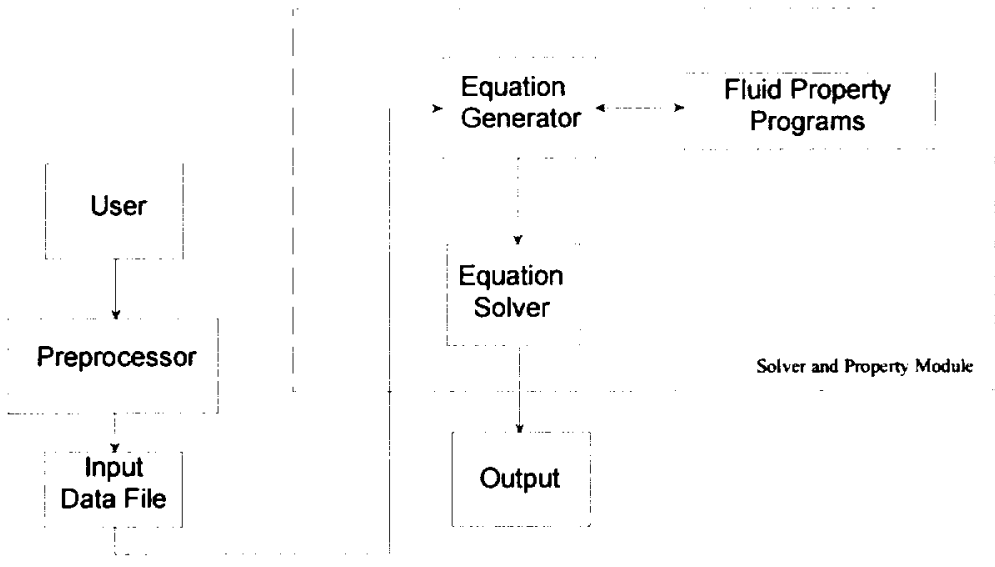

Figure 3. - GFSSP Process Flow Diagram 


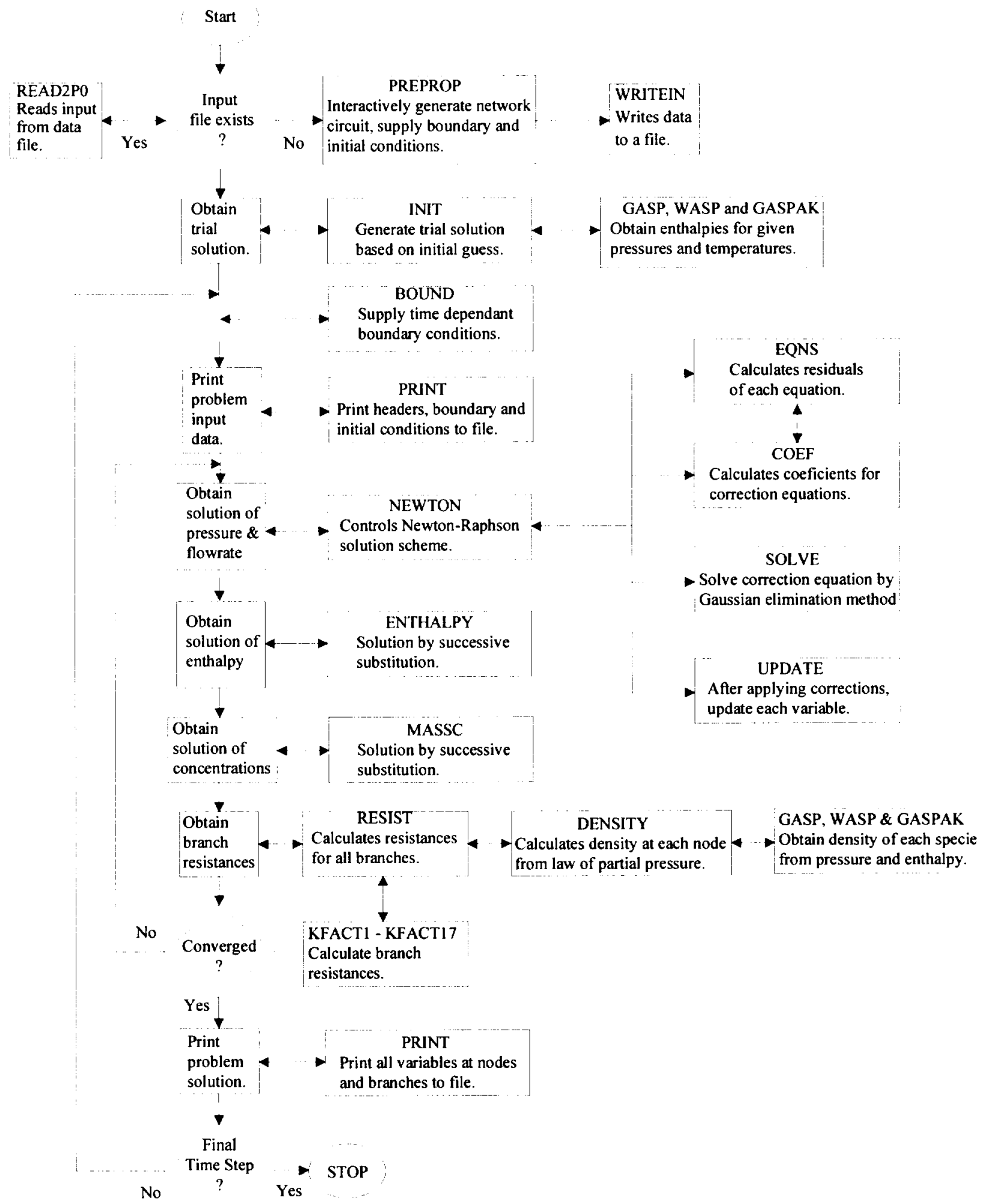

Figure 4. GFSSP Flowchart of Major Subroutines 


\section{RESULTS \& DISCUSSION}

The paper also describes comparison of steady state flow network simulation with the predictions of Hardy Cross method and unsteady flow simulation of tank blow down with an analytical solution. A typical water distribution network is shown in Figure 5 and Table 2.

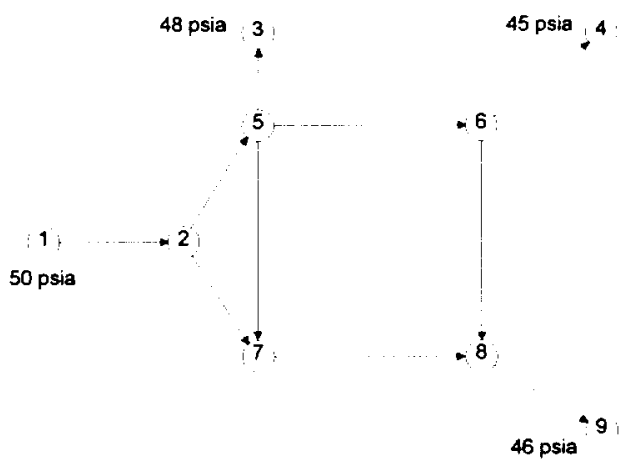

Figure 5 - Water Distribution Network Schematic

Table 2 - Water Distribution Network Branch Data

\begin{tabular}{|c|c|c|c|}
\hline Branch & Length(inches) & Diameter(inches) & $\begin{array}{c}\text { Roughness } \\
\text { Factor }\end{array}$ \\
\hline 12 & 120 & 6 & 0.0018 \\
\hline 25 & 2400 & 6 & 0.0018 \\
\hline 27 & 2400 & 5 & 0.0018 \\
\hline 57 & 1440 & 4 & 0.0018 \\
\hline 53 & 120 & 5 & 0.0018 \\
\hline 56 & 2400 & 4 & 0.0018 \\
\hline 64 & 120 & 4 & 0.0018 \\
\hline 68 & 1440 & 4 & 0.0018 \\
\hline 78 & 2400 & 4 & 0.0018 \\
\hline 89 & 120 & 5 & 0.0018 \\
\hline
\end{tabular}

Figure 6 shows a comparison between GFSSP and Hardy Cross predicted flowrates. The comparison appears reasonable considering the fact that Hardy Cross method assumes a constant friction factor in the branch while GFSSP computes the friction factor for each branch during every iteration. Therefore, as the flowrates change the friction factor also changes. 


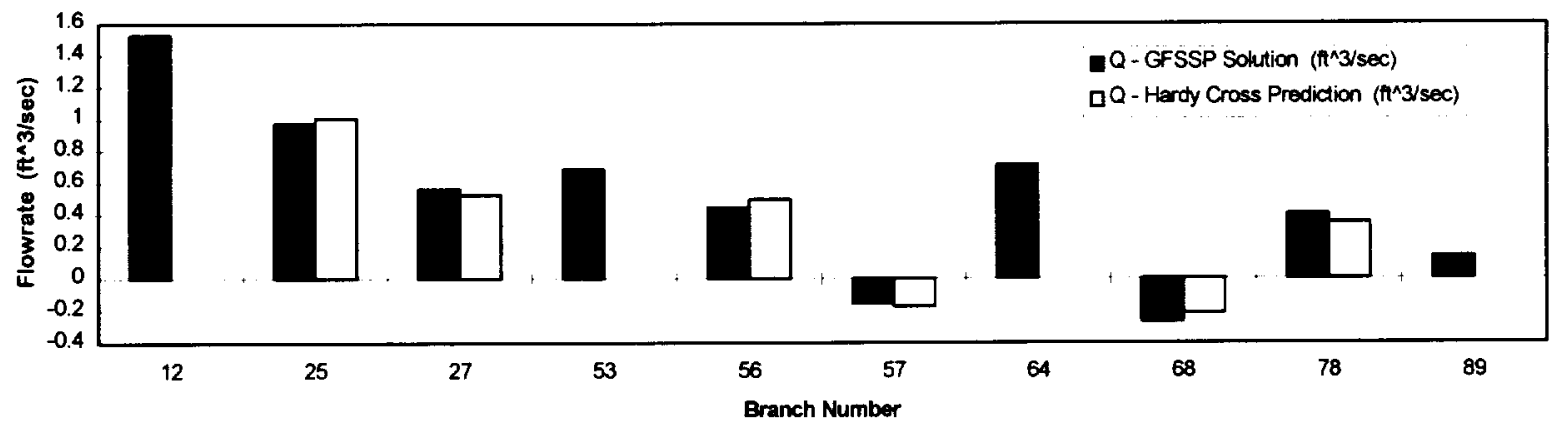

Figure 6. - A Flow Rate Comparison Between GFSSP and Hardy Cross Method Predictions

Blowdown of a Tank

Figure 7 shows a tank with an internal volume of $10 \mathrm{ft}^{3}$ containing nitrogen gas at a pressure and temperature of $100 \mathrm{psia}$ and $80^{\circ} \mathrm{F}$ respectively. The nitrogen is discharged into the atmosphere through an orifice with a 0.1 inch diameter until the pressure in the tank becomes 50 psia.

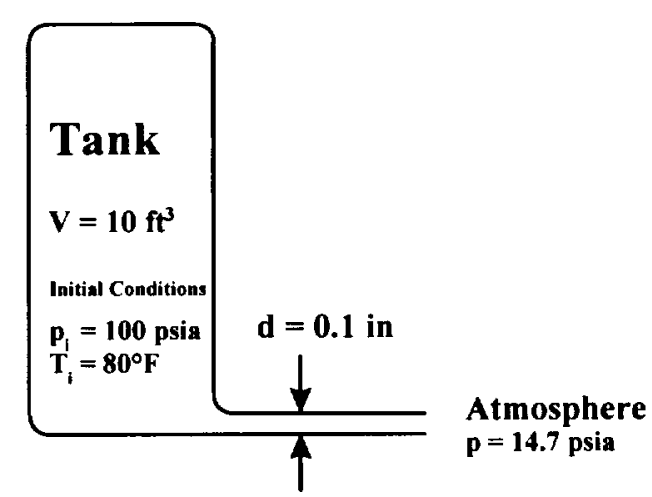

(a)

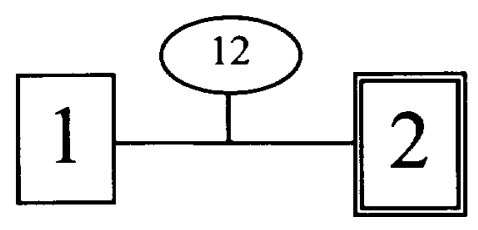

(b)

Figure 7. - Physical Schematic (a) and GFSSP Model (b) for Venting Nitrogen Tank

Analytical Solution:

The differential equation governing an isentropic blow down process can be written as:

$$
\left(\frac{p}{p_{i}}\right)^{(1-3 \gamma) / 2 \gamma} \frac{d\left(p / p_{i}\right)}{d \tau}=\frac{\gamma A}{\rho_{i} V} \sqrt{\gamma g_{c} p_{i} \rho_{i}}\left(\frac{2}{\gamma+1}\right)^{(\gamma+1) / 2(\gamma-1)}
$$

This is an initial value problem and the initial conditions are: 


$$
\tau=0, \frac{p}{p_{i}}=1
$$

The analytical solution for $p / p_{i}$ is given by Moody [23] as:

$$
\frac{p}{p_{i}}=\left[1+\left(\frac{\lambda-1}{2}\right)\left(\frac{2}{\gamma+1}\right)^{(\gamma+1) / 2(\gamma-1)} \sqrt{\frac{\gamma g_{c} p_{i}}{\rho_{i}}} \frac{A \tau}{V}\right]^{-2 \gamma /(\gamma-1)}
$$

The analytical and GFSSP solutions are compared in Figure 8. Excellent agreement was observed between the two solutions.

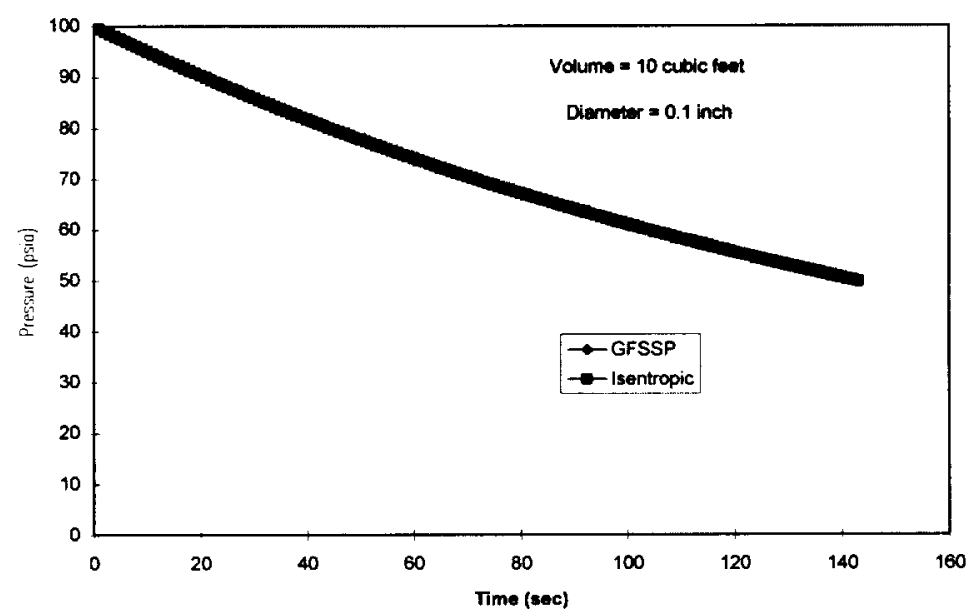

Figure 8 - Comparison of the Predicted Pressure History by GFSSP and the Analytical Solution

\section{REFERENCES}

1. Hardy Cross, "Analysis of Flow in Networks of Conduits or Conductors", Univ. Ill. Bull. 286, November 1936

2. Jeppson, Ronald W., "Analysis of Flow in Pipe Networks", Ann Arbor Science

3. Patankar, S. V., "Numerical Heat Transfer and Fluid Flow", Hemisphere Publishing Corp., Washington, D. C., 1980.

4. Hendricks, R. C., Baron, A. K., and Peller, I. C., "GASP - A Computer Code for Calculating the Thermodynamic and Transport Properties for Ten Fluids: Parahydrogen, Helium, Neon, Methane, Nitrogen, Carbon Monoxide, Oxygen, Fluorine, Argon, and Carbon Dioxide", NASA TN D-7808, February, 1975. 
5. Hendricks, R. C., Peller, I. C., and Baron, A. K., "WASP - A Flexible Fortran IV Computer Code for Calculating Water and Steam Properties", NASA TN D-7391, November, 1973.

6. Cryodata Inc., "User's Guide to GASPAK, Version 3.20", November, 1994.

1. 7.Moody, F. J., "Introduction to Unsteady Thermofluid Mechanics", John Wiley, 1990. 